\title{
An analytical study of drug utilization, disease progression, and adverse events among 165 COVID-19 patients
}

\author{
Feng Sun ${ }^{1,2 \#}$, Hao Kou ${ }^{1 \#}$, Shengfeng Wang ${ }^{2 \#, ~ Y u n ~ L u}{ }^{1}$, Houyu Zhao ${ }^{2}$, Wenjing Li ${ }^{1}$, Qingxin Zhou ${ }^{2}$, \\ Qiaoli Jiang ${ }^{1}$, Yinchu Cheng ${ }^{3}$, Kun Yang ${ }^{1}$, Lin Zhuo ${ }^{4}$, Yang $\mathrm{Xu}^{5}$, Dongfang Wu ${ }^{1}$, Siyan Zhan ${ }^{2,4}$, Hong Cheng ${ }^{1}$ \\ ${ }^{1}$ Department of Pharmacy, Zhongnan Hospital of Wuhan University, Wuhan, China; ${ }^{2}$ Department of Epidemiology and Biostatistics, School of \\ Public Health, Peking University, Beijing, China; ${ }^{3}$ Department of Pharmacy, Peking University Third Hospital, Beijing, China; ${ }^{4}$ Research Center \\ of Clinical Epidemiology, Peking University Third Hospital, Beijing, China; ${ }^{5}$ Department of Medical Epidemiology and Biostatistics, Karolinska \\ Institute, Stockholm, Sweden \\ Contributions: (I) Conception and design: F Sun, H Kou, S Wang, H Cheng, S Zhan; (II) Administrative support: Y Lu, W Li, Q Jiang, K Yang, Q \\ Zhou, L Zhuo; (III) Provision of study materials or patients: H Cheng, S Zhan; (IV) Collection and assembly of data: H Kou, Y Lu, W Li, Q Jiang, \\ K Yang, D Wu, H Cheng; (V) Data analysis and interpretation: F Sun, H Kou, S Wang, H Zhao, Q Zhou, Y Xu, Y Cheng, L Zhuo, H Cheng, S \\ Zhan; (VI) Manuscript writing: All authors; (VII) Final approval of manuscript: All authors. \\ "These authors contributed equally to this work. \\ Correspondence to: Hong Cheng, MD, PhD. Department of Pharmacy, Zhongnan Hospital of Wuhan University, 169 Donghu Road, Wuchang \\ District, Wuhan 430071, China. Email: chenghong@znhospital.cn; Siyan Zhan, MD, PhD. Department of Epidemiology and Biostatistics, School of \\ Public Health, Peking University, 38 Xueyuan Road, Haidian District, Beijing 100191, China. Email: siyan-zhan@bjmu.edu.cn.
}

Background: The coronavirus disease 2019 (COVID-19) epidemic has lasted for nearly 4 months by this study was conducted. We aimed to describe drug utilization, disease progression, and adverse drug events of COVID-19.

Methods: A retrospective, single-center case series study enrolled 165 consecutive hospitalized COVID-19 patients who were followed up until March 25, 2020, from a designated hospital in Wuhan. Patients were grouped by a baseline degree of severity: non-severe and severe. An analytical study of drug utilization, disease progression, and adverse events (AEs) of COVID-19 was conducted.

Results: Of the 165 COVID-19 cases, antivirals, antibacterials, glucocorticoids, and traditional Chinese medicine (TCM) were administered to $92.7 \%, 98.8 \%, 68.5 \%$, and $55.2 \%$ of patients, respectively. The total kinds of drugs administered to the severe subgroup [26, interquartile range (IQR) 18-39] were 11 more than the non-severe subgroup (15, IQR 10-24), regardless of comorbidities. The 2 most common combinations of medications in the 165 cases were 'antiviral therapy + glucocorticoids + TCM' (81, 49.1\%) and 'antiviral therapy + glucocorticoids' $(23,13.9 \%)$. Compared with non-severe cases, severe cases received more glucocorticoids ( $88.5 \%$ vs. $66.2 \%, \mathrm{P}=0.02)$, but less TCM (50.0\% vs. 63.3\%, $\mathrm{P}=0.20)$, and suffered a higher percentage of death $(34.6 \%$ vs. $7.2 \%, \mathrm{P}=0.001)$. At the end of the follow-up, $130(78.8 \%)$ patients had been discharged, and $24(14.5 \%)$ died. There were 13 patients $(7.9 \%)$ who had elevated liver enzymes, and 49 patients $(29.7 \%)$ presented with worsening kidney function during the follow-up.

Conclusions: Of the 165 COVID-19 patients, the fatality rate remained high (14.5\%). Drug utilization for COVID-19 was diverse and generally complied with the existing guidelines. Combination regimens containing antiviral drugs might be beneficial to assist COVID-19 recovery. Additionally, liver and kidney AEs should not be ignored.

Keywords: Novel coronavirus disease (COVID-19); drug utilization; disease progression; fatality; adverse events (AEs)

Submitted Jun 27, 2020. Accepted for publication Nov 20, 2020.

doi: 10.21037/atm-20-4960

View this article at: http://dx.doi.org/10.21037/atm-20-4960 


\section{Introduction}

The outbreak of coronavirus disease 2019 (COVID-19) has quickly swept across the world $(1,2)$, and has been declared a Public Health Emergency of International Concern (PHEIC) since January 30, $2020(3,4)$. As of April 20, 2020, 210 countries and territories worldwide have reported a total of $2,481,026$ confirmed cases and a death toll of 170,423. This situation already poses a serious global public health risk.

In the early stages of the outbreak, despite facing many challenges in understanding and treating COVID-19, especially a lack of specific antiviral agents, attempts at medication strategies had already been introduced into clinical practice by Chinese front-line physicians (5-9). To date, the $7^{\text {th }}$ updated version of the official diagnosis and treatment guidelines has already been published (10-14), and some achievements have been made in improving case fatality and enhancing the cure rate of COVID-19 patients. However, some concerns, including medication choices and combination and safety issues, have inevitably been raised (9,15-18). Previous studies have only described general epidemiological findings, clinical presentation, and clinical outcomes of COVID-19 patients (5-9,19-22). Furthermore, few of these studies have systematically characterized the drug utilization of COVID-19 patients. Therefore, our study's objectives were to give a full description of drug utilization, disease progression, and adverse drug events (ADEs) of COVID-19. We present the following article following the MDAR reporting checklist (available at http:// dx.doi.org/10.21037/atm-20-4960).

\section{Methods}

\section{Participants and data sources}

This retrospective, single-center case series study enrolled 165 consecutive COVID-19 patients initially hospitalized at Zhongnan Hospital of Wuhan University in Wuhan, China, from December 19, 2019, to February 2, 2020. All patients were followed up to March 25, 2020, and were $\geq 18$ years old and not diagnosed with bacterial pneumonia. Zhongnan Hospital is one of the major tertiary teaching hospitals in Wuhan, Hubei Province, and has been responsible for treating COVID-19 patients assigned by the government. According to the World Health Organization (WHO) interim guidance, all patients enrolled in this study were confirmed to be COVID-19 positive by viral test on admission (WHO) interim guidance $(23,24)$.

The participants' predefined information was extracted from electronic medical records (EMR), including demographics, treatment, and prognosis. A trained team of physicians and clinical pharmacists reviewed all data for accuracy and completeness. The research database was composed of 3 parts: (I) baseline characteristics, including demographics, COVID-19 contact history, underlying comorbidities; (II) diagnosis and treatment, including symptoms and signs, laboratory markers, chest computed tomographic (CT) scans, and medication (i.e., dosage, initial, and prescription and discontinuation date); (III) prognosis (death, recovery, or remained in hospital).

\section{Medications and outcomes}

We mainly focused on 9 classes of treatments according to the different versions of the guidelines for diagnosis and treatment of COVID-19 (Table S1): antivirals for systemic use [Anatomical Therapeutic Chemical (ATC) classification codes starting with J05], antibacterials for systemic use (J01), glucocorticoids for systemic use (H02AB), antimycotics for systemic use (J02), traditional Chinese medicines (TCM, identified by using drug name), general nutrients (V06), vasoactive drugs (C01DA, C01CA, and C04AB01), intestinal microecological regulators $(\mathrm{A} 07 \mathrm{~F})$, and immunoglobulins (J06BA). The total kinds of medications (according to generic names) used per person during hospitalization were also calculated-each prescription interval accumulated the total treatment duration for specific classes/kinds of drugs.

According to the guidelines, patient baseline condition severity was classified into 4 levels: mild, general, severe, and critically severe (12). The first 2 levels were further combined as the non-severe subgroup, and the latter 2 as the severe subgroup. Disease exacerbation was defined as the measure of disease condition getting worse at any time after admission.

Blood samples were tested for creatinine (reference value $<90 \mu \mathrm{mol} / \mathrm{L}$ ), alanine aminotransferase (ALT, reference value $<45 \mathrm{U} / \mathrm{L}$ ), and aspartate aminotransferase (AST, reference value $<40 \mathrm{U} / \mathrm{L})$. Urine samples were tested for albumin (normal sign with "negative"). The creatinine result was used in an equation with the patient's age, race, and sex to calculate the glomerular filtration rate (GFR, normal range of $\geq 90 \mathrm{~mL} / \mathrm{min} / 1.73 \mathrm{~m}^{2}$ ) (25). Laboratory abnormalities were used to define elevated serum aminotransferase levels and impaired renal function.

\section{Statistical analysis}

We first compared the baseline characteristics (including 
age, gender, occupation, etc.), drug utilization (including types of drugs, combination patterns, the number of the medications, total treatment duration, dosage, etc.), and potential adverse events (AEs) between the non-severe and severe subgroups. Furthermore, basic characteristics were compared between patients that had ever used or never used specific classes/kinds of drugs to explore the potentially influential factors for drug selection.

Frequency and percentages were described for categorical variables, and $\chi^{2}$ or Fisher's exact test was used for comparing the proportions in different subgroups. Median and interquartile range (IQR) were reported for the count and continuous variables, and the two-sample median test (26) was used for comparing medians of different subgroups. A two-sided $\mathrm{P}$ value of less than 0.05 was considered statistically significant. All analyses were performed using the SAS software (version 9.4) and R software (version 3.6.2).

The study was conducted following the Declaration of Helsinki (as revised in 2013). The Institutional Ethics Board of Zhongnan Hospital of Wuhan University approved this study (No. 2020014). Written informed consent was waived for emerging infectious diseases.

\section{Results}

\section{Baseline features}

The study included 165 COVID-19 patients. The median age was 55 years (IQR, 42-66; range, 22-96 years), and $84(50.9 \%)$ were men. Of these patients, $26(15.8 \%)$ were in the severe subgroup, and $139(84.2 \%)$ were in the non-severe subgroup at admission. Compared with the non-severe subgroup (Table 1), the severe patients were approximately 13 years older, with a higher proportion of comorbidities ( $84.6 \%$ vs. $44.6 \%$ ). The most common comorbidities were hypertension $(24.8 \%)$, cardiovascular disease $(9.7 \%)$, diabetes $(7.3 \%)$, and cancer $(4.8 \%)$. A nonsignificant difference was detected in either sex, contact history, or other clinical features between the 2 groups, except that the severe subgroup had more frequent onsets of dyspnea or shortness of breath $(23.1 \%$ vs. $4.3 \%$ for the non-severe subgroup) (Table S2).

\section{Overall drug utilization}

Among the 165 cases, antivirals (75.8\% for oseltamivir, $43.0 \%$ for $\alpha$-interferon, $13.9 \%$ for lopinavir/ritonavir), antibacterials, glucocorticoids, general nutrients, and TCM were received by $92.7 \%, 98.8 \%, 69.7 \%, 77.0 \%$, and $61.2 \%$ of patients, respectively (Table 2). The combinations of the medications were quite diverse (Figure S1), and the top 4 medication combinations were antivirals combined with glucocorticoids and TCM (81, 49.1\%), antivirals combined with glucocorticoids $(23,13.9 \%)$, only antivirals $(27,16.4 \%)$, and antivirals combined with TCM (22, $13.3 \%)$ without considering other coexisting medications (Table S3). A median of 17 (IQR, 10-29) kinds of drugs were prescribed to each patient. Patients with and without comorbidities took a median of 21 (IQR, 15-40) and 12 (IQR, 8-19) kinds of drugs, respectively, and the difference was statistically significant $(\mathrm{P}<0.001)$ (Figure $1 A$ and Table S3). Most patients received only 1 kind of antiviral drug (IQR, 1-2), and only 5 patients took more than 3 kinds of antiviral drugs during hospitalization.

The median duration of antivirals was 8 days (IQR, 6-12), with $30.9 \%$ of patients taking antivirals longer than 10 days. Antibacterials and glucocorticoids were treated with a median of 12 days (IQR, 9-18) and 7 days (IQR, 4-12), respectively (Table 2). Regarding the doses of antivirals and glucocorticoids, the single-dose administrations mostly followed the guidelines (Table S4). Also, patients with comorbidities were less likely to receive TCM, whereas patients who were older or with more comorbidities were more likely to be administered other medications (Tables S5-S12).

\section{Drug utilization differences between severity groups}

Compared with non-severe cases, more severe cases received glucocorticoids ( $88.5 \%$ vs. $66.2 \%, \mathrm{P}=0.02)$ and vasoactive drugs (50.0\% vs. 19.4\%, $\mathrm{P}<0.001)$, but received less TCM $(50.0 \%$ vs. $63.3 \%, \mathrm{P}=0.20)$. The total kinds of drugs administered to the severe subgroup (27, IQR 18-41) was 12 more than the non-severe subgroup (15, IQR 10-27) regardless of comorbidities (Figure $1 A, \mathrm{P}<0.001$ ). Severe cases were more likely to take a higher single dose ( 5 million $\mathrm{U}$ ) of $\alpha$-interferon, a longer glucocorticoid duration, or a shorter immunoglobulin treatment. All other features, in terms of duration or singledose administrations, were not significantly different between the 2 severity groups (Table 2 and Table S4).

\section{Patterns of disease progression}

By March 25, 130 (78.8\%) of the 165 patients had been discharged. Of all 165 patients, 24 (14.5\%) patients had died, while the rest of the patients were still in the hospital 
Table 1 Baseline characteristics of 165 patients with coronavirus disease 2019 (COVID-19)

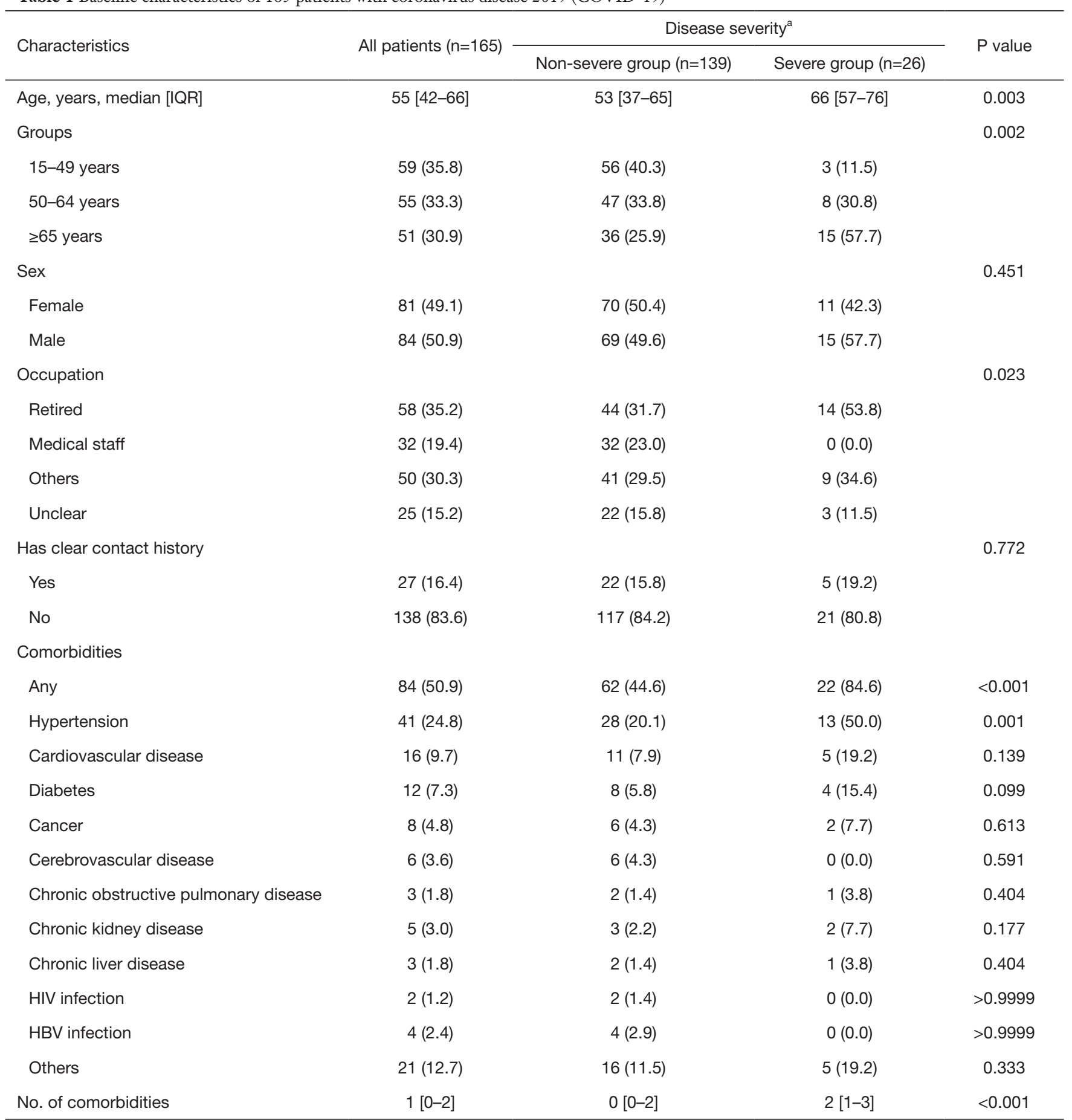

Data are presented as no. (\%) or median [IQR]. ${ }^{\text {, }}$, the patient's baseline condition was classified into 4 levels according to the guidelines: mild, general, severe, and critically severe, respectively. The first 2 levels were further classified as the non-severe subgroup, and the latter 2 as the severe subgroup. HIV, human immunodeficiency virus; HBV, hepatitis B virus; IQR, interquartile range. 
Table 2 Drug utilization and their duration for 165 patients with coronavirus disease 2019 (COVID-19)

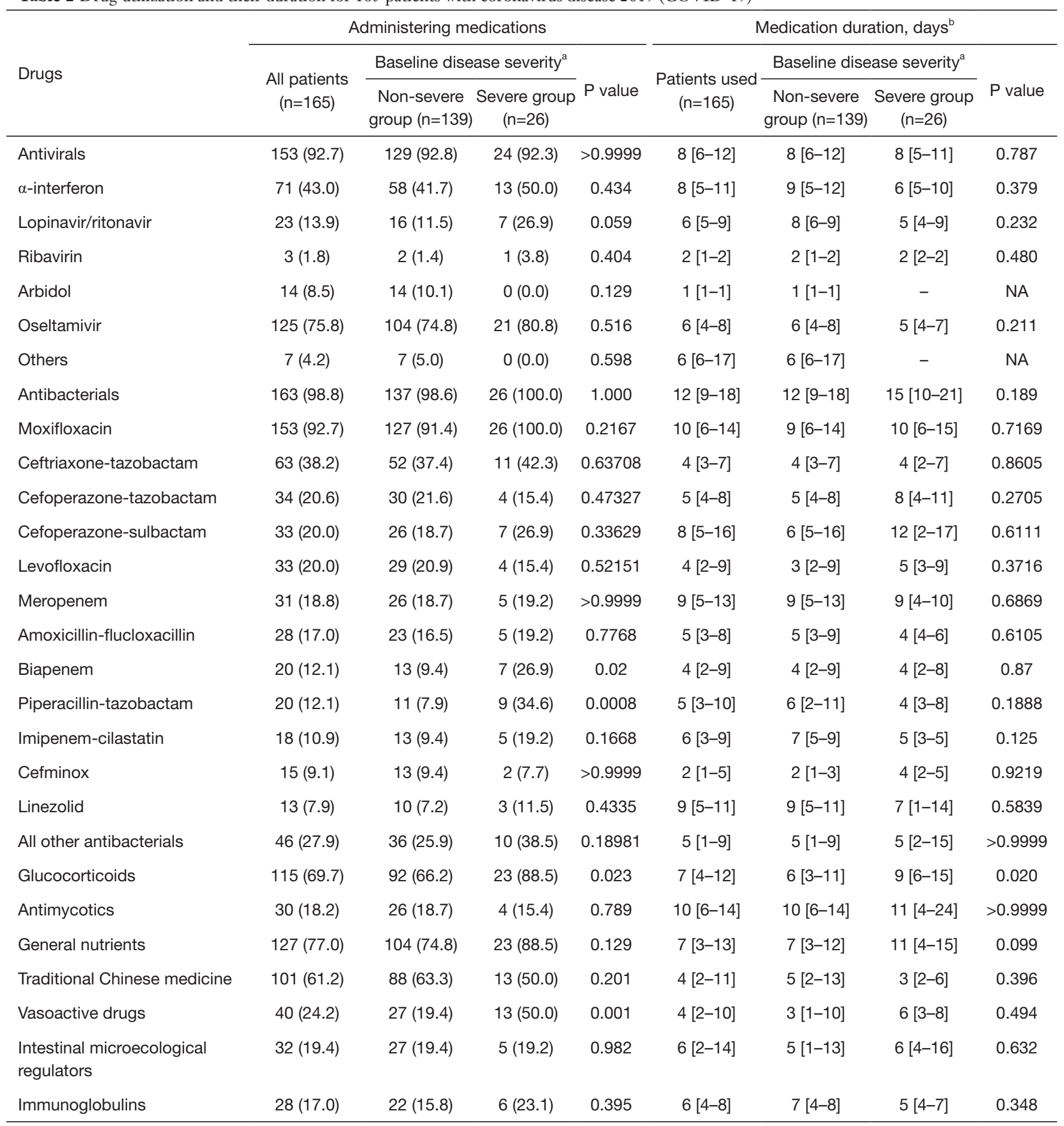

Data are presented as no. (\%) or median [IQR]. Medications include antivirals [Anatomical Therapeutic Chemical (ATC) classification codes starting with J05], antibacterials (J01), glucocorticoids (H02AB), antimycotics (J02), general nutrients (V06), traditional Chinese medicine (TCM, identified using drug name), vasoactive drugs (C01DA, C01CA, C04AB01), intestinal microecological regulators (A07F), and immunoglobulins (J06BA). ${ }^{a}$, the patient's baseline condition was classified into 4 levels according to the guidelines: mild, general, severe, and critically severe, respectively. The first 2 levels were further classified as the non-severe subgroup, and the latter 2 as the severe subgroup. " , the total treatment duration for specific classes/kinds of drugs was accumulated by each prescription interval. "-" means that none of severe patients were treated with that class of medication. NA, not applicable; IQR, interquartile range. 


\section{A severity 审Non-severe 㚔 Severe 审 Overall}
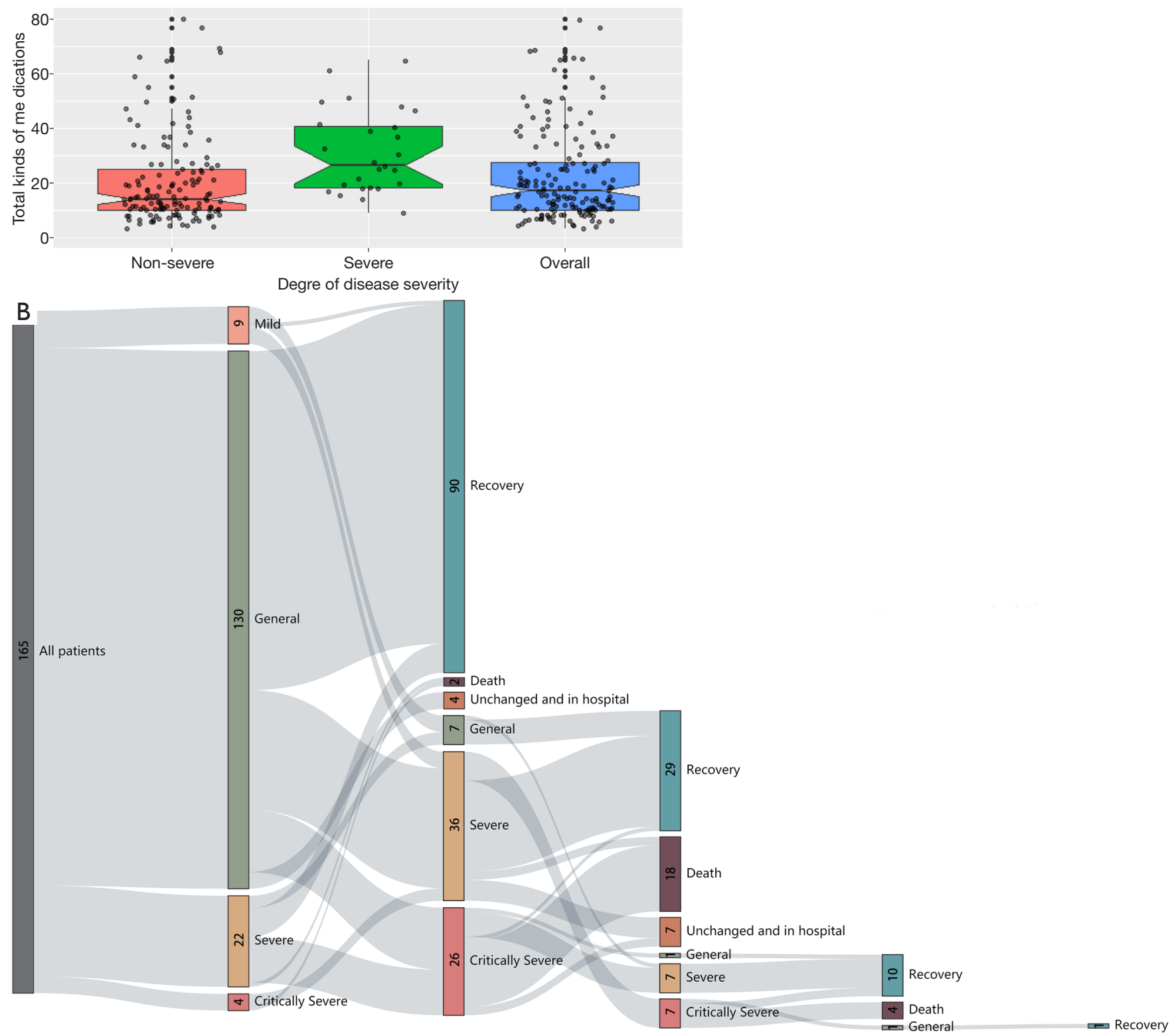

Figure 1 The total kinds of medications and disease progression for 165 patients with coronavirus disease 2019 (COVID-19). (A) The total kinds of medications grouped by disease severity and comorbidities. Total kinds of medications refer to the medications (generic names) per person used during the whole hospitalization. Antivirals were defined as Anatomical Therapeutic Chemical (ATC) classification codes starting with J05. (B) The disease progression for 165 patients since baseline. The patient's baseline condition was classified into 4 levels according to the guidelines "Diagnostic and treatment protocol for Novel Coronavirus Pneumonia (trial version 5)": mild, general, severe, and critically severe, respectively.

or transferred to other hospitals. Figure $1 B$ shows the cumulative outcomes of the patient cohort. It can be seen that $11.1 \%(1 / 9), 12.3 \%(16 / 130), 36.4 \%(8 / 22)$, and $25.0 \%$ $(1 / 4)$ of the patients progressed to a worse condition or even death for those with baseline mild, general, severe, and critically severe levels, respectively. Compared with the non-severe subgroup, the patients in the severe subgroup experienced a significantly higher percentage of death 
Table 3 Outcomes of death or disease exacerbation after admission for patients using different drugs

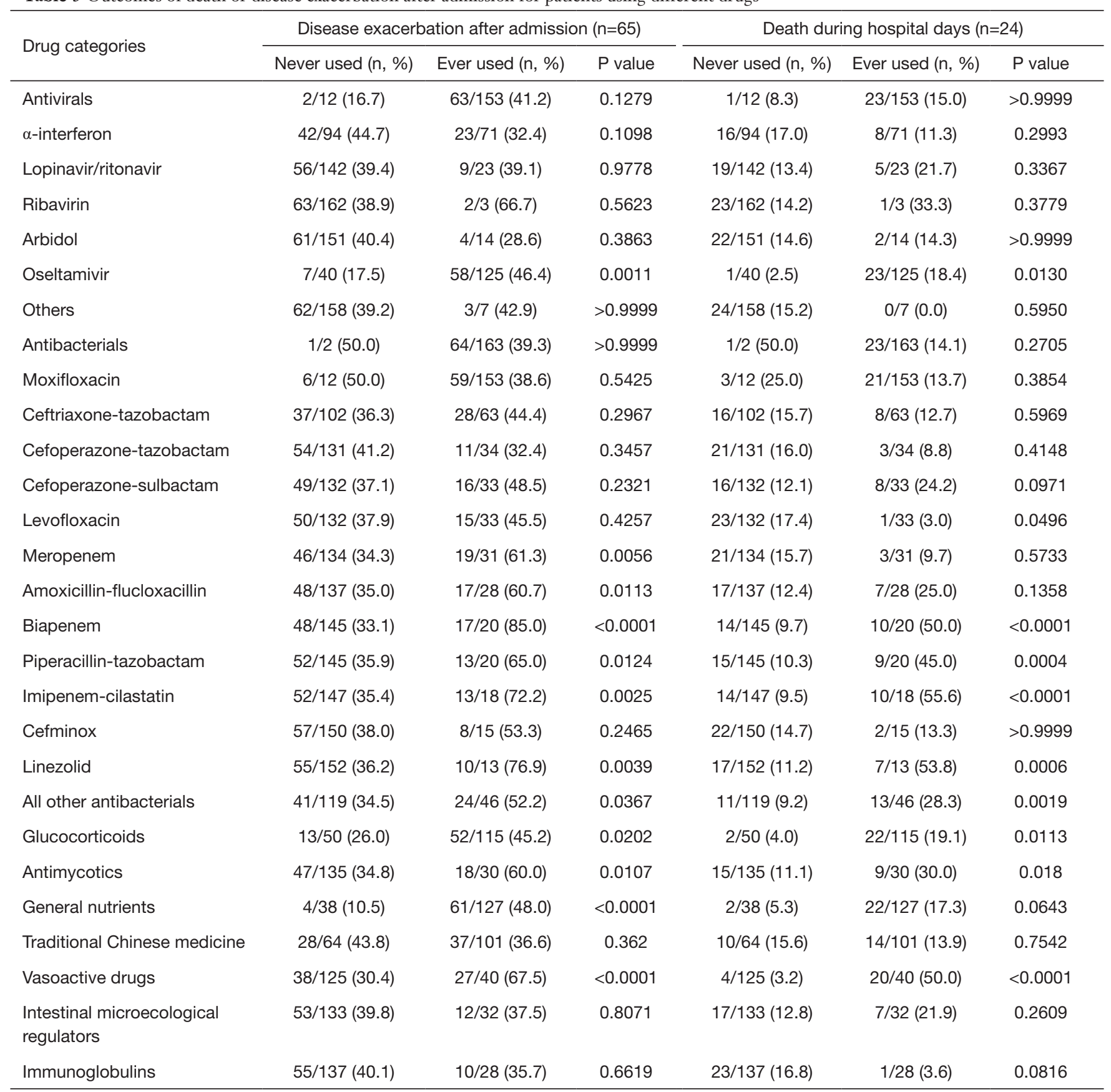

Data are presented as no. (\%).

(34.6\% vs. $7.2 \%, \mathrm{P}=0.001)$ and a shorter period from hospital admission to ICU admission (median, 3 vs. 6 days; IQR, $0-5$ vs. 4-8 days, $\mathrm{P}<0.001)$. For the 24 death cases, a total of 16 patients $(66.7 \%)$ deteriorated $(7,29.2 \%)$ or even died $(9,70.8 \%)$ within the first 7 days of hospitalization (Figure S2). There were no differences observed in the rate of disease exacerbation or death during hospitalization between patients who ever used antivirals, antibacterials, TCM, intestinal microecological regulators, and immunoglobulins (Table 3). In the patients who had disease exacerbation or died during hospitalization, 'antivirals + glucocorticoids + TCM' was the most common medication 
Table 4 Outcomes of death or disease exacerbation after admission for patients using different drug combinations

\begin{tabular}{|c|c|c|c|c|c|c|c|c|}
\hline Medication combinations & \multicolumn{4}{|c|}{ Disease progression } & \multicolumn{4}{|c|}{ Disease mortality } \\
\hline Antivirals + glucocorticoids + TCM & $81(49.1)$ & $42(51.9)$ & $39(48.1)$ & 0.0169 & $81(49.1)$ & $61(75.3)$ & $20(24.7)$ & $<0.0001$ \\
\hline Antivirals + glucocorticoids & $23(13.9)$ & $12(52.2)$ & $11(47.8)$ & & $23(13.9)$ & $22(95.7)$ & $1(4.3)$ & \\
\hline Antivirals + TCM & $22(13.3)$ & $19(86.4)$ & $3(13.6)$ & & $22(13.3)$ & $22(100.0)$ & $0(0.0)$ & \\
\hline Others (all without antivirals) & $12(7.3)$ & $10(83.3)$ & $2(16.7)$ & & $12(7.3)$ & $11(91.7)$ & $1(8.3)$ & \\
\hline
\end{tabular}

Data are presented as $\mathrm{n}(\%)$. TCM, traditional Chinese medicine.

combination (Table 4).

\section{Safety assessment}

Two senior clinical pharmacists independently evaluated the association between AEs in patients and their medication regimens. The basic criteria for distinguishing AEs with COVID-19 related presentation included excluding any patient who had liver or kidney injury history and presented with abnormal liver or kidney function on admission. There were $53(32.1 \%)$ cases of AEs, of which 13 patients (7.9\%) had elevated liver enzymes, 49 patients $(29.7 \%)$ presented with worsening kidney function, and 9 patients had both. A total of 157 tested patients without liver injury history presented an average AST level of 34.0 (22.0-61.0) U/L in blood. The number of cases with elevated blood AST enzymes was $7.9 \%(11 / 139)$ and $15.4 \%(4 / 26)$ in severe and non-severe patients, respectively, and the difference in the average level between the 2 groups was statistically significant $(\mathrm{P}=0.002)$. A total of 159 patients were subjected to ALT tests, and the average level was 30.0 (17.0-68.0) U/L. As for patients with abnormal blood ALT levels, the number of cases was also $7.9 \%(11 / 139)$ and $15.4 \%$ (4/26) for the 2 groups, respectively, and the difference in average level between the 2 groups was also statistically significant $(\mathrm{P}=0.003)$. Also, 45 (31.5\%), 25 (42.4\%), and 30 (66.7\%) patients presented with worsening kidney function as determined by the 3 indicators of creatinine, EGFR, and urine protein, respectively, among 143, 59, and 45 patients tested without chronic kidney disease (CKD) history (Table 5).

\section{Discussion}

To our knowledge, this is one of the earliest studies to describe the detailed patterns of medication, disease progression, as well as safety issues for hospitalized patients with COVID-19. We also found that the prescriptions were diverse in practice, and most of the medications were prescribed considering the patient's characteristics, including disease severity, age, comorbidities, and AEs. However, the almost universal use of antibacterials might have caused a significant proportion of liver injury or kidney injury. Our findings provide important clues for further explorations, especially regarding treatment timing and safety issues.

The clinical features of patients with COVID-19 in our study were consistent with 4 recent reports, with fever as the predominant symptom (6-9). Compared with the nonsevere patients, the severe subgroup cases were significantly older and were more likely to have comorbidities, and these findings were also compatible with 2 previous studies $(6,9)$. Nearly all patients in this study received antibacterials, $92.7 \%$ received antivirals, and $69.7 \%$ received glucocorticoids. These results were following 3 recent investigations conducted in Wuhan (7-9), but were significantly higher than the 2 latest reports outside Wuhan $(5,6)$. This inconsistency might be because patients outside Wuhan in the previous studies were at least 8 years younger, with less severe disease and comorbidities $(5,6,8,9)$. An unsurprising finding of our study is that clinicians tried several drugs and even more drug combinations as potential pharmaceutical options against COVID-19, even within a single hospital. The diverse medication regimens might be because no specific treatment has been recommended for COVID-19 until now, and the evolution and revisions to the government guidelines (trial) for the diagnosis and treatment of COVID-19 are constant (10-13,27). Over 200 studies have already been registered on either ClinicalTrials.gov or Chictr.org, to test medications that fight other viruses (e.g., flu and HIV), TCM, stem cells, steroids, and plasma treatment. However, we have to accept that all treatment explorations require processes of a certain 
Table 5 Test results and liver and kidney adverse events of COVID-19 patients

\begin{tabular}{|c|c|c|c|c|c|c|c|c|}
\hline Test results & \multicolumn{4}{|c|}{ Non-severe group $(n=139)$} & \multicolumn{4}{|c|}{ Severe group $(n=26)$} \\
\hline \multicolumn{9}{|l|}{ AST (U/L) } \\
\hline Sample size & 134 & 11 & 123 & & 23 & 4 & 19 & \\
\hline Median (IQR) & $\begin{array}{c}31.5 \\
(22.0-54.0)\end{array}$ & $\begin{array}{c}134.0 \\
(48.0-180.0)\end{array}$ & $\begin{array}{c}30.0 \\
(21.0-45.0)\end{array}$ & $<0.0001$ & $\begin{array}{c}68.0 \\
(48.0-81.0)\end{array}$ & $\begin{array}{c}187.0 \\
(167.5-204.0)\end{array}$ & $\begin{array}{c}63.0 \\
(36.0-71.0)\end{array}$ & 0.002 \\
\hline Sample size & 134 & 11 & 123 & & 25 & 4 & 21 & \\
\hline Median (IQR) & $\begin{array}{c}26.0 \\
(16.0-58.0)\end{array}$ & $\begin{array}{c}184.0 \\
(88.0-279.0)\end{array}$ & $\begin{array}{c}24.0 \\
(15.0-47.0)\end{array}$ & $<0.0001$ & $\begin{array}{c}59.0 \\
(30.0-91.0)\end{array}$ & $\begin{array}{c}172.0 \\
(133.5-254.0)\end{array}$ & $\begin{array}{c}55.0 \\
(27.0-79.0)\end{array}$ & 0.003 \\
\hline \multicolumn{9}{|l|}{ CRE } \\
\hline \multicolumn{9}{|l|}{ EGFR } \\
\hline Sample size & 50 & 20 & 30 & & 9 & 5 & 4 & \\
\hline Median (IQR) & $\begin{array}{c}107.0 \\
(83.1-119.7)\end{array}$ & $\begin{array}{c}78.5 \\
(69.6-89.5)\end{array}$ & $\begin{array}{c}114.1 \\
(105.4-122.0)\end{array}$ & $<0.0001$ & $\begin{array}{c}111.6 \\
(80.4-115.7)\end{array}$ & $\begin{array}{c}80.4 \\
(71.4-120.6)\end{array}$ & $\begin{array}{c}112.0 \\
(110.7-114.0)\end{array}$ & 0.713 \\
\hline \multicolumn{9}{|l|}{ Upro } \\
\hline Sample size & 33 & 21 & 12 & & 12 & 9 & 3 & \\
\hline- & 13 & $1(7.7)$ & $12(92.3)$ & $<0.0001$ & 3 & $0(0.0)$ & $3(100.0)$ & 0.005 \\
\hline
\end{tabular}

Data are presented as no. (\%) or median (IQR). AST, aspartate aminotransferase; ALT, alanine aminotransferase; CRE, creatinine; EGFR, estimated glomerular filtration rate; Upro, urine protein; IQR, interquartile range.

time frame (28) and that the current state of chaos will inevitably last for some time. Therefore, to sum up, it is critical to further investigate drug utilization and potential experiences promptly like our study in order to provide real-world evidence for clinical decision-makers.

A higher proportion and a longer duration of glucocorticoids are worth noting in this study. Current WHO guidance and several researchers have recommended that corticosteroids should not be used due to COVID-19induced lung injury or shock $(17,23)$. In contrast, an expert consensus statement developed by the Chinese Thoracic Society on February 11 points out that corticosteroids should not be abandoned in treating COVID-19 due to inconclusive clinical evidence (19). According to this statement, the dose should be low-to-moderate $(\leq 0.5-1 \mathrm{mg} / \mathrm{kg}$ per day methylprednisolone or equivalent), and the duration should be short ( $\leq 7$ days). Our study indicated that almost all the single doses of corticosteroids were already consistent with this statement. This might due to this hospital having developed rapid guidelines before January 29 (27), with a weak recommendation that 40 to $80 \mathrm{mg}$ of methylprednisolone per day could be considered. However, approximately half of the patients were treated with corticosteroids for more than 7 days, which was more serious in severe patients. This finding was consistent with 2 previous studies completed before developing the expert 
consensus statement (8). The main reason is most likely due to the rapid development of this hospital's guidelines, which did not mention the duration. The current national guidelines recommend that glucocorticoids should be restricted within 3-5 days $(6,10-13)$ if needed. Considering corticosteroid treatment is a 'double-edged sword' (19), our finding is worthy of front-line physicians' and researchers' attention.

Another phenomenon that should be noted is the almost universal empirical antibacterial treatment. This result was in agreement with 3 studies conducted in Wuhan $(6,7,9)$ but was twice the level of the latest study outside Wuhan (5). The difference in the distribution of age and comorbidities between $\mathrm{Xu}$ et al.'s study and ours might partially explain this gap in the antibacterial usage rate (5). Also, inadequate supplies of specific detection kits in Wuhan during late January and early February 2020 brought about difficulties in making rapid etiology diagnoses of COVID-19 in patients on admission, resulting in requests for empirical antibacterial treatment to rule out a bacterial infection, and consequently increased antibacterial usage rates. All COVID-19 treatment statements in China emphasized to avoid inappropriate use of antibacterials, especially the combination of broad-spectrum antibacterials (10-13,27). In this study, we observed possible AEs in the liver and kidney at a common level (over 5\%).

Interestingly, these safety signals have also been reported by some antibacterial instructions and previous studies (29-31). The kidneys' potential harm was also well summarized in previous studies for amoxicillin (30) and cloxacillin sodium (31). The widespread use of antibacterials, together with multiple drugs, should alert clinicians to pay attention to the potential ADEs (32).

Our study focused on drug utilization and disease progression from real-world data. Some limitations should be noted in this study. First, only 165 patients from a single hospital were included, and $3.0 \%$ of patients were still hospitalized at the time of database locking. However, despite this, the results of this study permitted an early assessment. Second, with the limited number of nonsevere cases, only age, sex, and the number of comorbidities were taken into consideration, and additional confounders might still have existed. Although almost all antivirals and antibacterials (the most common treatments in our study) were not over-the-counter medications in China (33), it is unknown what percentage of patients obtained the drugs from outpatient services. Therefore, the percentage of prehospitalization medications should be further considered in future investigations.

\section{Conclusions}

In summary, the drug utilization for hospitalized patients with COVID-19 was diverse and generally complied with China's existing guidelines. Also, AEs should not be ignored in the process of drug prescriptions. Given our preliminary investigation, there is a need for multicenter research with larger sample size and longer follow-up period in the future in order to promote a more solid basis for medication recommendations.

\section{Acknowledgments}

Funding: This study was supported by the National Key Technology R\&D Program of China (grant number 2020YFC0840800), National Natural Science Foundation of China (grant number 81973146), Fundamental Research Funds for the Central Universities (grant number 2042020kf1019), Special Research Fund of PKUHSC for Prevention and Control of COVID-19 (grant number BMU2020HKYZX010) and the Fundamental Research Funds for the Central Universities. The funders had no role in study design, data collection, data analysis, data interpretation, writing of the manuscript, and decision to submit.

\section{Footnote}

Reporting Checklist: The authors have completed the MDAR checklist. Available at http://dx.doi.org/10.21037/atm-204960

Data Sharing Statement: Available at http://dx.doi. org/10.21037/atm-20-4960

Peer Review File: Available at http://dx.doi.org/10.21037/ atm-20-4960

Conflicts of Interest: All authors have completed the ICMJE uniform disclosure form (available at http://dx.doi. org/10.21037/atm-20-4960). Dr. SZ reports grants from National Key Technology R\&D Program of China, grants from National Natural Science Foundation of China, grants from Special Project for Major Infectious Diseases of Peking University Health Science Center, during the conduct of the study. Dr. YC reports grants from Fundamental 
Research Funds for the Central Universities, during the conduct of the study. The other authors have no conflicts of interest to declare.

Ethical Statement: The authors are accountable for all aspects of the work in ensuring that questions related to the accuracy or integrity of any part of the work are appropriately investigated and resolved. The corresponding author had access to all data in the study and had final responsibility for the decision to submit for publication. The study was conducted in accordance with the Declaration of Helsinki (as revised in 2013). This study was approved by the Institutional Ethics Board of Zhongnan Hospital of Wuhan University (No. 2020014). Written informed consent was waived for emerging infectious diseases.

Open Access Statement: This is an Open Access article distributed in accordance with the Creative Commons Attribution-NonCommercial-NoDerivs 4.0 International License (CC BY-NC-ND 4.0), which permits the noncommercial replication and distribution of the article with the strict proviso that no changes or edits are made and the original work is properly cited (including links to both the formal publication through the relevant DOI and the license). See: https://creativecommons.org/licenses/by-nc-nd/4.0/.

\section{References}

1. Lu R, Zhao X, Li J, et al. Genomic characterisation and epidemiology of 2019 novel coronavirus: implications for virus origins and receptor binding. Lancet 2020;395:565-74.

2. Zhu N, Zhang D, Wang $W$, et al. A Novel Coronavirus from Patients with Pneumonia in China, 2019. N Engl J Med 2020;382:727-33.

3. Phelan AL, Katz R, Gostin LO. The Novel Coronavirus Originating in Wuhan, China: Challenges for Global Health Governance. JAMA 2020;323:709-10.

4. Wang C, Horby PW, Hayden FG, et al. A novel coronavirus outbreak of global health concern. Lancet 2020;395:470-3

5. Xu XW, Wu XX, Jiang XG, et al. Clinical findings in a group of patients infected with the 2019 novel coronavirus (SARS-Cov-2) outside of Wuhan, China: retrospective case series. BMJ 2020;368:m606.

6. Guan WJ, Ni ZY, Hu Y, et al. Clinical Characteristics of Coronavirus Disease 2019 in China. N Engl J Med 2020;382:1708-20.

7. Huang C, Wang Y, Li X, et al. Clinical features of patients infected with 2019 novel coronavirus in Wuhan, China. Lancet 2020;395:497-506.

8. Chen N, Zhou M, Dong X, et al. Epidemiological and clinical characteristics of 99 cases of 2019 novel coronavirus pneumonia in Wuhan, China: a descriptive study. Lancet 2020;395:507-13.

9. Wang D, Hu B, Hu C, et al. Clinical Characteristics of 138 Hospitalized Patients With 2019 Novel CoronavirusInfected Pneumonia in Wuhan, China. JAMA 2020;323:1061-9.

10. General Office of the National Health Commission. General Office of Administration of Traditional Chinese Medicine. Diagnostic and treatment protocol for Novel Coronavirus Pneumonia (trial third version). January 22, 2020. Available online: http://www.gov.cn/zhengce/ zhengceku/2020-01/23/content_5471832.htm. Accessed February 12, 2020.

11. General Office of the National Health Commission. General Office of Administration of Traditional Chinese Medicine. Diagnostic and treatment protocol for Novel Coronavirus Pneumonia (trial fourth version). January 27, 2020. Available online: http://www.gov.cn/zhengce/ zhengceku/2020-01/28/content_5472673.htm. Accessed February 12, 2020.

12. General Office of the National Health Commission. General Office of Administration of Traditional Chinese Medicine. Diagnostic and treatment protocol for Novel Coronavirus Pneumonia (trial fifth version). February 4, 2020. Available online: http://www.nhc.gov.cn/yzygj/s765 3p/202002/3b09b894ac9b4204a79db5b8912d4440.shtml. Accessed February 12, 2020.

13. General Office of the National Health Commission. General Office of Administration of Traditional Chinese Medicine. Diagnostic and treatment protocol for Novel Coronavirus Pneumonia (trial sixth version). February 18, 2020. Available online: http://www.nhc.gov.cn/jkj/s35 77/202003/4856d5b0458141fa9f376853224d41d7.shtml. Accessed February 18, 2020.

14. General Office of the National Health Commission. General Office of Administration of Traditional Chinese Medicine. Diagnostic and treatment protocol for Novel Coronavirus Pneumonia (trial seventh version). March 3, 2020. Available online: http://www.nhc.gov.cn/cms-search/ xxgk/getManuscriptXxgk.htm?id=318683 cbfaee4191aee29 cd774b19d8d. Accessed March 6, 2020.

15. Supe S, Kohse F, Gembardt F, et al. Therapeutic time window for angiotensin-(1-7) in acute lung injury. $\mathrm{Br} \mathrm{J}$ Pharmacol 2016;173:1618-28. 
16. Richardson P, Griffin I, Tucker C, et al. Baricitinib as potential treatment for 2019-nCoV acute respiratory disease. Lancet 2020;395:e30-e31.

17. Russell CD, Millar JE, Baillie JK. Clinical evidence does not support corticosteroid treatment for 2019-nCoV lung injury. Lancet 2020;395:473-5.

18. Wang FS, Zhang C. What to do next to control the 2019nCoV epidemic? Lancet 2020;395:391-3.

19. Shang L, Zhao J, Hu Y, et al. On the use of corticosteroids for 2019-nCoV pneumonia. Lancet 2020;395:683-4.

20. Yang X, Yu Y, Xu J, et al. Clinical course and outcomes of critically ill patients with SARS-CoV-2 pneumonia in Wuhan, China: a single-centered, retrospective, observational study. Lancet Respir Med 2020;8:475-81.

21. Chen H, Guo J, Wang C, et al. Clinical characteristics and intrauterine vertical transmission potential of COVID-19 infection in nine pregnant women: a retrospective review of medical records. Lancet 2020;395:809-15.

22. Chang D, Lin M, Wei L, et al. Epidemiologic and Clinical Characteristics of Novel Coronavirus Infections Involving 13 Patients Outside Wuhan, China. JAMA 2020;323:1092-3.

23. World Health Organization. Clinical management of severe acute respiratory infection when Novel coronavirus (2019$\mathrm{nCoV}$ ) infection is suspected: Interim Guidance. January 28, 2020. Available online: https://www.who.int/docs/defaultsource/coronaviruse/clinical-management-of-novel-cov. pdf?sfvrsn=bc7da517_2. Accessed January 31, 2020.

24. Deng SQ, Peng HJ. Characteristics of and Public Health Responses to the Coronavirus Disease 2019 Outbreak in China. J Clin Med 2020;9:575.

25. Levey AS, Stevens LA, Schmid CH, et al. A new equation

Cite this article as: Sun F, Kou H, Wang S, Lu Y, Zhao H, Li W, Zhou Q, Jiang Q, Cheng Y, Yang K, Zhuo L, Xu Y, Wu D, Zhan S, Cheng H. An analytical study of drug utilization, disease progression, and adverse events among 165 COVID-19 patients. Ann Transl Med 2021;9(4):306. doi: 10.21037/atm-20-4960 to estimate glomerular filtration rate. Ann Intern Med 2009;150:604-12.

26. Wolfe DA. Two-stage two-sample median test. Technometrics 1977;19:495-501.

27. Jin Y, Cai L, Cheng Z, et al. A rapid advice guideline for the diagnosis and treatment of 2019 novel coronavirus (2019-nCoV) infected pneumonia (standard version). Mil Med Res 2020;7:4.

28. Horton R. Offline: 2019-nCoV Outbreak-Early Lessons. Lancet 2020;395:322.

29. Haverkamp W, Kruesmann F, Fritsch A, et al. Update on the cardiac safety of moxifloxacin. Curr Drug Saf 2012;7:149-63.

30. Guzman-Limon M, Molony D. In older patients with UTIs, trimethoprim vs amoxicillin was linked to higher risk for AKI at $14 \mathrm{~d}$. Annals of internal medicine 2018;168:JC58.

31. Lavergne A, Vigneau C, Polard E, et al. Acute Kidney Injury During Treatment With High-Dose Cloxacillin: A Report of 23 Cases and Literature Review. Int J Antimicrob Agents 2018;52:344-9.

32. Fan Z, Chen L, Li J, et al. Clinical Features of COVID19-Related Liver Functional Abnormality. Clin Gastroenterol Hepatol 2020;18:1561-6.

33. General Office of the National Health Commission. Notice of the general office of China National Health Commission on the continuous management of the clinical application of antibiotics. March 29, 2019. Available online: http://www.nhc.gov.cn/yzygj/s7659/201903/1d487 eb7b7c74abc9fcb104f8b0905f2.shtml. Accessed February 5,2020 . 
Table S2 Clinical features of 165 patients with coronavirus disease 2019 (COVID-19)

\begin{tabular}{|c|c|c|c|c|}
\hline Clinical features & All patients $(n=165)$ & \multicolumn{2}{|c|}{ Baseline disease severity $^{a}$} & $P$ value \\
\hline \multicolumn{5}{|l|}{ Symptoms and signs } \\
\hline Fever & $125(75.8)$ & $102(73.4)$ & $23(88.5)$ & 0.100 \\
\hline Fatigue & $20(12.1)$ & $18(12.9)$ & $2(7.7)$ & 0.743 \\
\hline Anorexia & $3(1.8)$ & $2(1.4)$ & $1(3.8)$ & 0.404 \\
\hline Myalgia & $7(4.2)$ & $7(5.0)$ & $0(0.0)$ & 0.598 \\
\hline Dyspnea or shortness of breath & $12(7.3)$ & $6(4.3)$ & $6(23.1)$ & 0.004 \\
\hline Chill & $2(1.2)$ & $2(1.4)$ & $0(0.0)$ & 1.000 \\
\hline Diarrhea or abdominal pain & $9(5.5)$ & $8(5.8)$ & $1(3.8)$ & $>0.9999$ \\
\hline Nausea or vomiting & $5(3.0)$ & $4(2.9)$ & $1(3.8)$ & 0.581 \\
\hline Dizziness or headache & $4(2.4)$ & $4(2.9)$ & $0(0.0)$ & $>0.9999$ \\
\hline Nasal congestion & $2(1.2)$ & $1(0.7)$ & $1(3.8)$ & 0.291 \\
\hline Enlargement of lymph nodes & $1(0.6)$ & $1(0.7)$ & $0(0.0)$ & $>0.9999$ \\
\hline No. of Symptoms and signs & $1(1-2)$ & $1(1-2)$ & $1(1-2)$ & 0.300 \\
\hline Days of fever & $10(5-14)$ & $9(5-13)$ & $15(10-24)$ & 0.007 \\
\hline \multicolumn{5}{|l|}{ Abnormalities on chest CT } \\
\hline
\end{tabular}

a, the patient's baseline condition was classified into four levels according to the guidelines: mild, general, severe, critical severe, respectively, the first two levels were further classified as a non-severe subgroup and the latter two as a severe subgroup. Data are presented as No. (\%) or median (IQR). 
Table S3 The combination and the number of the medications for 165 patients with coronavirus disease 2019 (COVID-19)

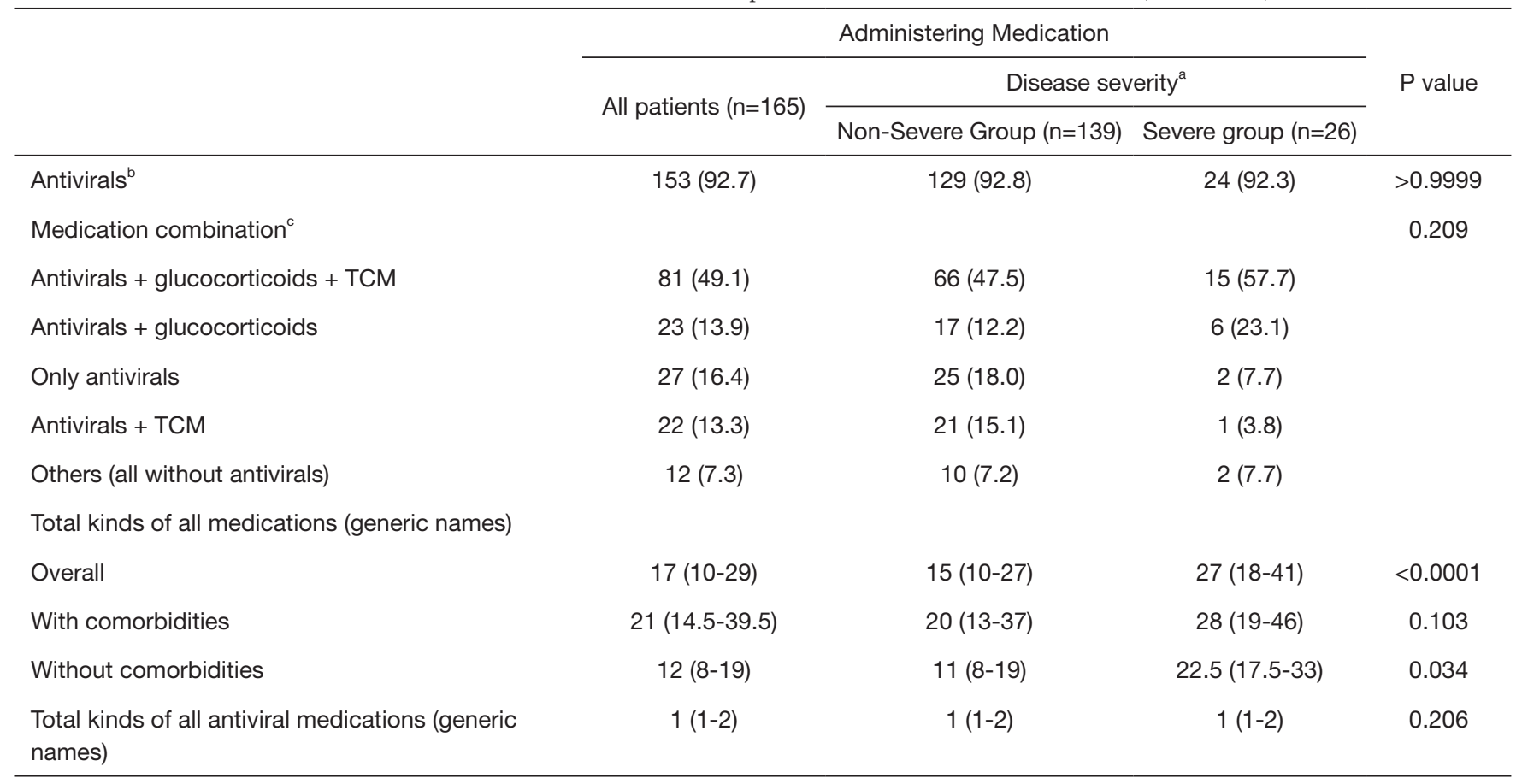

Data are presented as No. (\%) or median (IQR). ${ }^{\text {, }}$, the patient's baseline condition was classified into four levels according to the guidelines: mild, general, severe, critical severe, respectively, the first two levels were further classified as a non-severe subgroup and the latter two as a severe subgroup. ${ }^{b}$, antivirals was defined as Anatomical Therapeutic Chemical (ATC) classification codes started with J05, glucocorticoids (H02AB), traditional Chinese medicine (TCM, identified using drug name). ${ }^{\circ}$, medication combination analysis were concentrated on antivirals, glucocorticoids and TCM (traditional Chinese medicine) without considering other coexisting medications. IQR, interquartile range. 
Table S4 Dose distribution of antivirals and glucocorticoids for patients with coronavirus disease 2019 (COVID-19)

$$
\text { All patients }(n=165) \frac{\text { Baseline disease severity }^{a}}{\text { Non-severe group }(n=139) \text { Severe group }(n=26)} \quad \text { P value }
$$

Antivirals $^{b}$

$\alpha$-interferon

No. of its prescription

3 million $U$

5 million $U$

Others (power, etc.)

Lopinavir/litonavir

No. of its prescription

$2 \cdot 00 \mathrm{co}$

$400 \cdot 00 \mathrm{co}$

Others (tablet, etc.)

Arbidol

No. of its prescription

$200.00 \mathrm{mg}$

Others (dispersible tablet, etc.)

Oseltamivir

No. of its prescription

$150.00 \mathrm{mg}$

$75.00 \mathrm{mg}$

Capsule

Others (Granules)

Glucocorticoids ${ }^{c}$

Hexadecadrol

No. of its prescription

$3.00 \mathrm{mg}$

$5.00 \mathrm{mg}$

Others $(7.50 \mathrm{mg}, 20.00 \mathrm{mg}$, or hydroacupuncture)

Methylprednisolon

No. of its prescription

$20.00 \mathrm{mg}$

$40.00 \mathrm{mg}$

$60.00 \mathrm{mg}$

$80.00 \mathrm{mg}$

Others (power, etc.)

Other dosage

$$
\begin{gathered}
107(100.0) \\
79(73.8) \\
24(22.4) \\
4(3.7)
\end{gathered}
$$$$
92(86.0)
$$$$
73 \text { (79.3) }
$$$$
15(14.0)
$$$$
6(40.0)
$$$$
15 \text { (16.3) }
$$$$
9(60.0)
$$$$
4(4.3)
$$$$
0(0.0)
$$

$35(100.0)$

$27(77.1)$

8 (22.9)

$20(74.1)$

27 (77.1)

6 (22.2)

$0(0.0)$

1 (3.7)

1 (12.5)

2 (5.7)

$20(100.0)$

20 (100.0)

$0(0.0)$

1 (5.0)

19 (95.0)

$0(0.0)$

1 (5.0)

$0(0.0)$

$419(100.0)$
$16(3.8)$
$324(77.3)$
$77(18.4)$
$2(0.5)$

361 (86.2)

58 (13.8)

$13(3.6)$

3 (5.2)

279 (77.3)

45 (77.6)

67 (18.6)

$10(17.2)$

2 (0.6)

$0(0.0)$

0.237

0.002

(237

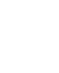

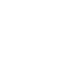


Table S5 Baseline features of all patients with coronavirus disease 2019 (COVID-19) who started treatment with or without antivirals

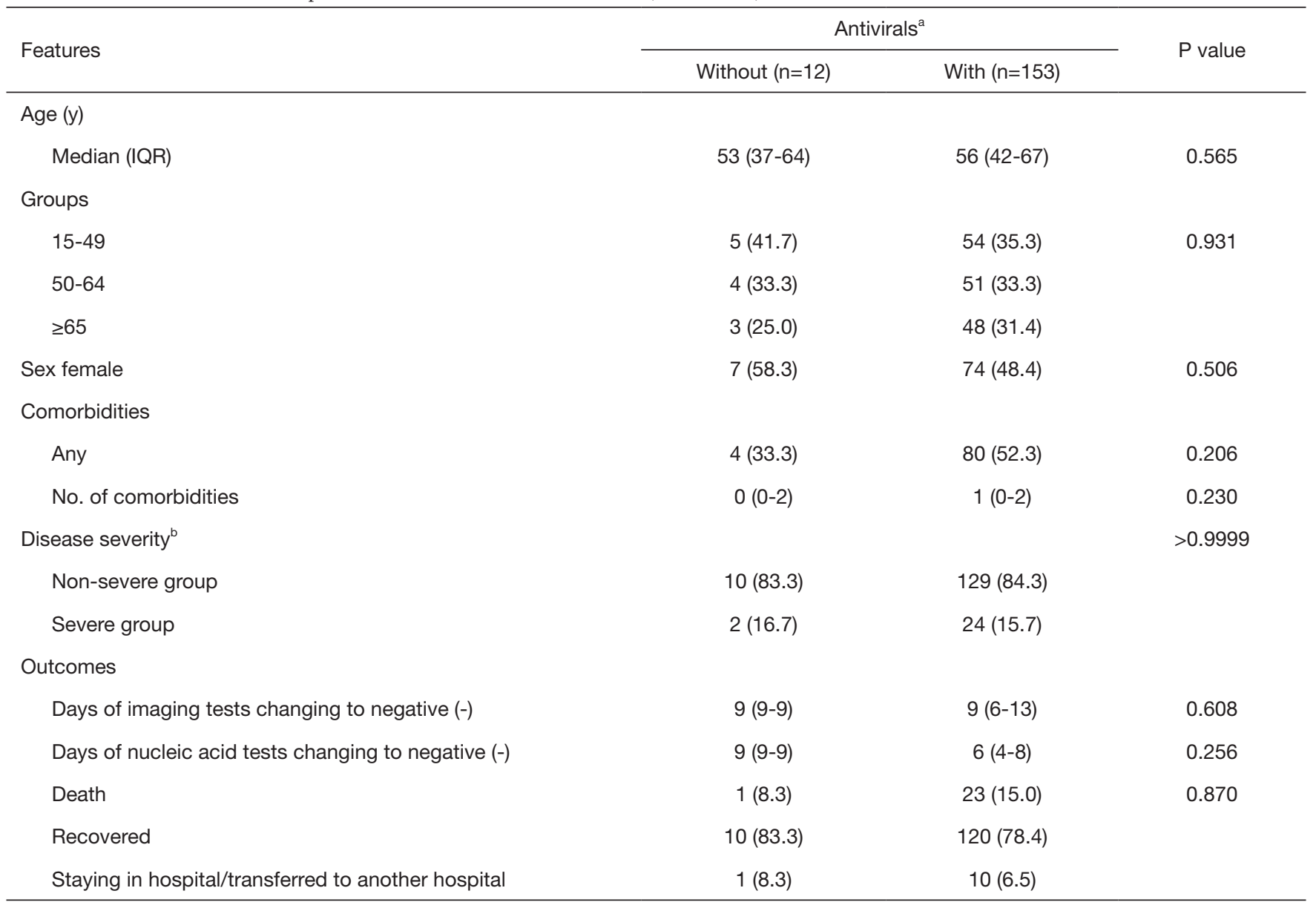

Data are presented as No. (\%) or median (IQR). ${ }^{\text {, }}$, antivirals was defined as Anatomical Therapeutic Chemical (ATC) classification codes started with J05. ${ }^{\text {b }}$, The patient's baseline condition was classified into four levels according to the guidelines: mild, general, severe, critical severe, respectively, the first two levels were further classified as a non-severe subgroup and the latter two as a severe subgroup. IQR, interquartile range. 
Table S6 Baseline features of all patients with coronavirus disease 2019 (COVID-19) who started treatment with or without glucocorticoids

\begin{tabular}{|c|c|c|c|}
\hline \multirow{2}{*}{ Features } & \multicolumn{2}{|c|}{ Glucocorticoids $^{a}$} & \multirow{2}{*}{$P$ value } \\
\hline & Without $(n=52)$ & With $(n=113)$ & \\
\hline \multicolumn{4}{|l|}{ Age (y) } \\
\hline Median (IQR) & $54(37-61)$ & $58(42-70)$ & 0.194 \\
\hline \multicolumn{4}{|l|}{ Groups } \\
\hline $50-64$ & $21(42.0)$ & $34(29.6)$ & \\
\hline$\geq 65$ & $10(20.0)$ & $41(35.7)$ & \\
\hline Sex female & $31(62.0)$ & $50(43.5)$ & 0.029 \\
\hline \multicolumn{4}{|l|}{ Comorbidities } \\
\hline Non-Severe group & $47(94.0)$ & $92(80.0)$ & 0.023 \\
\hline Severe group & $3(6.0)$ & $23(20.0)$ & \\
\hline \multicolumn{4}{|l|}{ Outcomes } \\
\hline Days of imaging tests changing to negative (-) & $8(4-11)$ & $10(8-14)$ & 0.058 \\
\hline Days of nucleic acid tests changing to negative (-) & $5(2-5)$ & $7(5-9)$ & 0.042 \\
\hline Death & $2(4.0)$ & $22(19.1)$ & 0.020 \\
\hline Recovered & $46(92.0)$ & $84(73.0)$ & \\
\hline
\end{tabular}

Data are presented as No. (\%) or median (IQR). ${ }^{\text {, }}$ glucocorticoids was defined as Anatomical Therapeutic Chemical (ATC) classification codes started with H02AB. " , the patient's baseline condition was classified into four levels according to the guidelines: mild, general, severe, critical severe, respectively, the first two levels were further classified as a non-severe subgroup and the latter two as a severe subgroup. IQR, interquartile range. 
Table S7 Baseline features of all patients with coronavirus disease 2019 (COVID-19) who started treatment with or without antimycotics

\begin{tabular}{|c|c|c|c|}
\hline Features & \multicolumn{2}{|c|}{ Antimycotics $^{a}$} & $P$ value \\
\hline \multicolumn{4}{|l|}{ Age (y) } \\
\hline Median (IQR) & $54(39-65)$ & $67(50-80)$ & 0.004 \\
\hline \multicolumn{4}{|l|}{ Groups } \\
\hline $50-64$ & $49(36.3)$ & $6(20.0)$ & \\
\hline$\geq 65$ & $34(25.2)$ & $17(56.7)$ & \\
\hline Sex female & $66(48.9)$ & $15(50.0)$ & 0.912 \\
\hline \multicolumn{4}{|l|}{ Comorbidities } \\
\hline Non-severe group & $113(83.7)$ & $26(86.7)$ & 0.789 \\
\hline Severe group & $22(16.3)$ & $4(13.3)$ & \\
\hline \multicolumn{4}{|l|}{ Outcomes } \\
\hline Days of imaging tests changing to negative (-) & $9(6-12)$ & $14(7-20)$ & 0.974 \\
\hline Days of nucleic acid tests changing to negative (-) & $6(4-8)$ & $5(2-11)$ & 0.562 \\
\hline Death & $15(11.1)$ & $9(30.0)$ & $<0.0001$ \\
\hline Recovered & $116(85.9)$ & $14(46.7)$ & \\
\hline
\end{tabular}

Data are presented as No. (\%) or median (IQR). a , antimycotics was defined as Anatomical Therapeutic Chemical (ATC) classification codes started with $\mathrm{J} 02 .^{\text {b }}$, the patient's baseline condition was classified into four levels according to the guidelines: mild, general, severe, critical severe, respectively, the first two levels were further classified as a non-severe subgroup and the latter two as a severe subgroup. $I Q R$, interquartile range. 
Table S8 Baseline features of all patients with coronavirus disease 2019 (COVID-19) who started treatment with or without general nutrients

\begin{tabular}{|c|c|c|c|}
\hline \multirow{2}{*}{ Features } & \multicolumn{2}{|c|}{ General nutrients $^{a}$} & \multirow{2}{*}{$P$ value } \\
\hline & Without $(n=39)$ & With $(n=126)$ & \\
\hline \multicolumn{4}{|l|}{ Age (y) } \\
\hline Median (IQR) & $51(34-65)$ & $57(43-67)$ & 0.487 \\
\hline \multicolumn{4}{|l|}{ Groups } \\
\hline $50-64$ & $10(26.3)$ & $45(35.4)$ & \\
\hline$\geq 65$ & $10(26.3)$ & $41(32.3)$ & \\
\hline Sex female & $24(63.2)$ & $57(44.9)$ & 0.048 \\
\hline \multicolumn{4}{|l|}{ Comorbidities } \\
\hline Non-severe group & $35(92.1)$ & $104(81.9)$ & 0.129 \\
\hline Severe group & $3(7.9)$ & $23(18.1)$ & \\
\hline \multicolumn{4}{|l|}{ Outcomes } \\
\hline Days of imaging tests changing to negative (-) & $9(8-12)$ & $9(6-13)$ & 0.914 \\
\hline Days of nucleic acid tests changing to negative (-) & $6(4-7)$ & $6(4-9)$ & 0.894 \\
\hline Death & $2(5.3)$ & $22(17.3)$ & 0.073 \\
\hline Recovered & $35(92.1)$ & $95(74.8)$ & \\
\hline
\end{tabular}

Data are presented as No. (\%) or median (IQR). ${ }^{\text {, }}$ general nutrients was defined as Anatomical Therapeutic Chemical (ATC) classification codes started with V06. ${ }^{b}$, the patient's baseline condition was classified into four levels according to the guidelines: mild, general, severe, critical severe, respectively, the first two levels were further classified as a non-severe subgroup and the latter two as a severe subgroup. $I Q R$, interquartile range. 
Table S9 Baseline features of all patients with coronavirus disease 2019 (COVID-19) who started treatment with or without traditional Chinese medicine

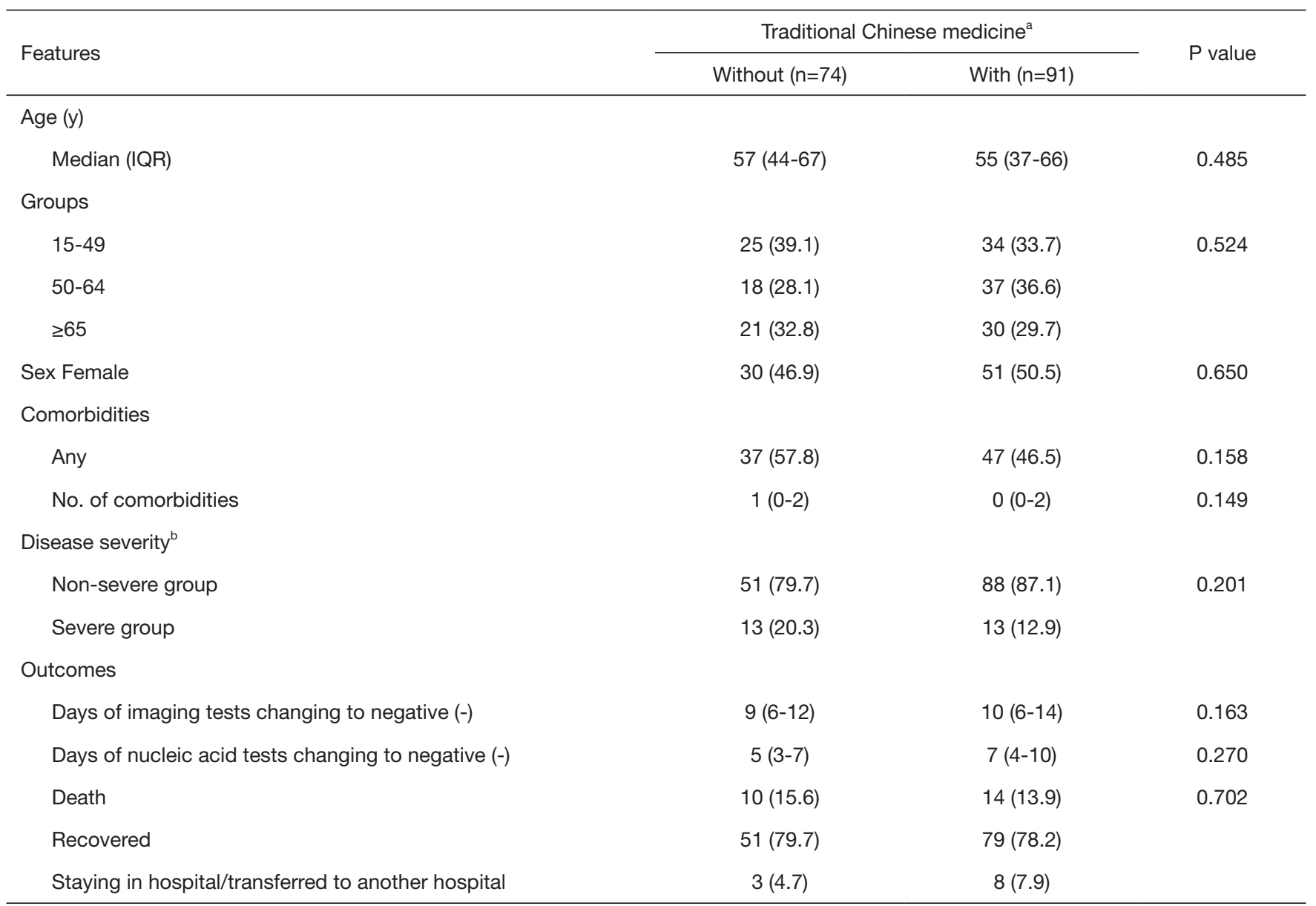

Data are presented as No. (\%) or median (IQR). a, traditional Chinese medicine was defined as Anatomical Therapeutic Chemical (ATC) classification codes started with TCM, identified using drug name. ${ }^{b}$, the patient's baseline condition was classified into four levels according to the guidelines: mild, general, severe, critical severe, respectively, the first two levels were further classified as a non-severe subgroup and the latter two as a severe subgroup. IQR, interquartile range. 
Table S10 Baseline features of all patients with coronavirus disease 2019 (COVID-19) who started treatment with or without vasoactive drugs

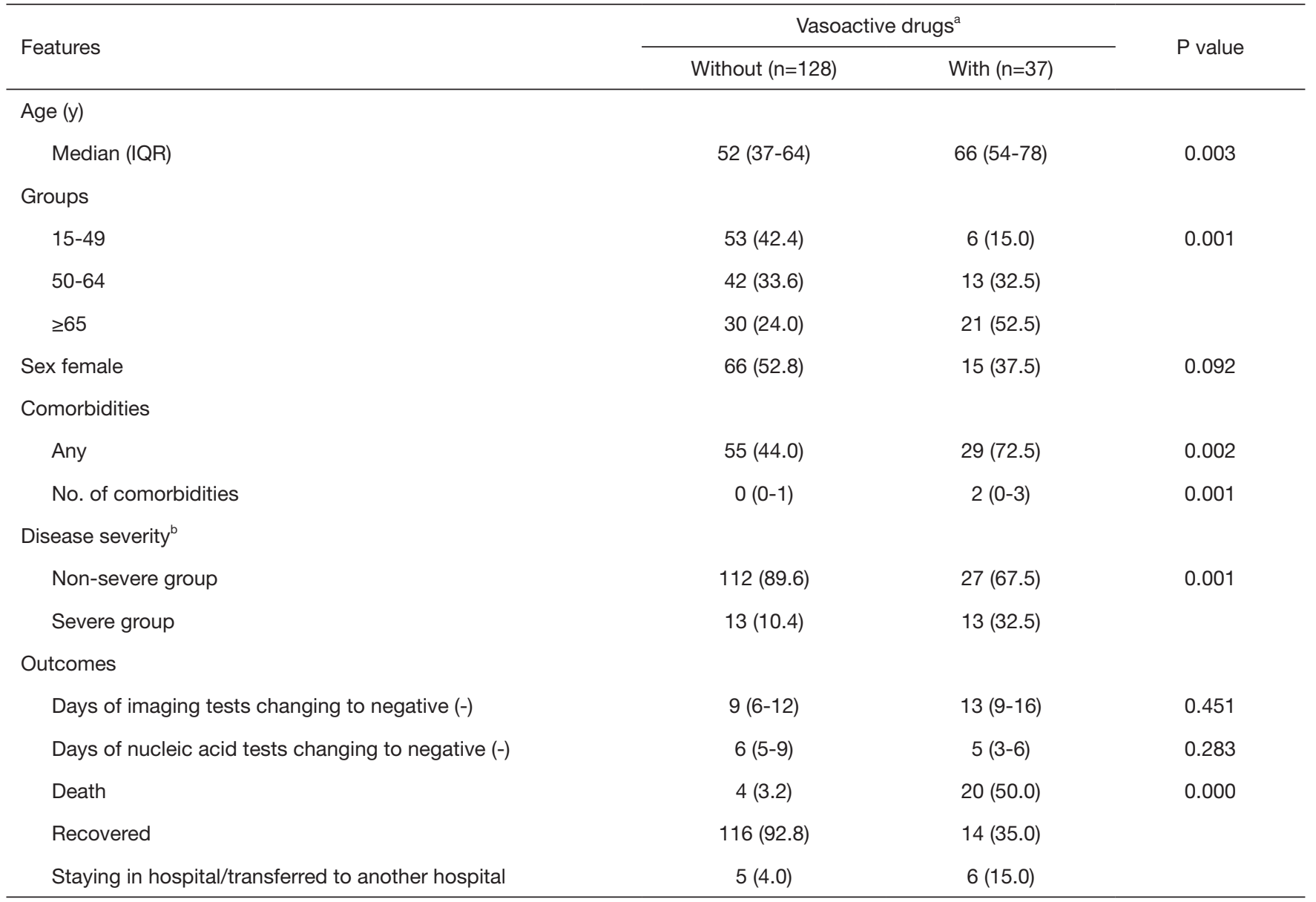

Data are presented as No. (\%) or median (IQR). ${ }^{\text {, }}$, vasoactive drugs was defined as Anatomical Therapeutic Chemical (ATC) classification codes started with C01DA, C01CA, C04AB01. ${ }^{b}$, the patient's baseline condition was classified into four levels according to the guidelines: mild, general, severe, critical severe, respectively, the first two levels were further classified as a non-severe subgroup and the latter two as a severe subgroup. IQR, interquartile range. 
Table S11 Baseline features of all patients with coronavirus disease 2019 (COVID-19) who started treatment with or without intestinal microecological regulators

\begin{tabular}{|c|c|c|c|}
\hline Features & \multicolumn{2}{|c|}{ Intestinal microecological regulators ${ }^{\mathrm{a}}$} & $P$ value \\
\hline \multicolumn{4}{|l|}{ Age $(y)$} \\
\hline Median (IQR) & $54(39-65)$ & $65(48-77)$ & 0.045 \\
\hline \multicolumn{4}{|l|}{ Groups } \\
\hline $50-64$ & $47(35.3)$ & $8(25.0)$ & \\
\hline$\geq 65$ & $35(26.3)$ & $16(50.0)$ & \\
\hline Sex female & $62(46.6)$ & $19(59.4)$ & 0.195 \\
\hline \multicolumn{4}{|l|}{ Comorbidities } \\
\hline \multicolumn{4}{|l|}{ Disease severity ${ }^{b}$} \\
\hline Non-severe group & $112(84.2)$ & $27(84.4)$ & 0.982 \\
\hline Severe group & $21(15.8)$ & $5(15.6)$ & \\
\hline \multicolumn{4}{|l|}{ Outcomes } \\
\hline Days of imaging tests changing to negative (-) & $9(7-13)$ & $12(4-20)$ & 0.501 \\
\hline Days of nucleic acid tests changing to negative (-) & $6(4-7)$ & $8(4-12)$ & 0.926 \\
\hline Death & $17(12.8)$ & $7(21.9)$ & 0.004 \\
\hline Recovered & $111(83.5)$ & $19(59.4)$ & \\
\hline
\end{tabular}

Data are presented as No. (\%) or median (IQR). ${ }^{\text {a }}$, intestinal microecological regulators was defined as Anatomical Therapeutic Chemical (ATC) classification codes started with A07F. ${ }^{b}$, the patient's baseline condition was classified into four levels according to the guidelines: mild, general, severe, critical severe, respectively, the first two levels were further classified as a non-severe subgroup and the latter two as a severe subgroup. IQR, interquartile range. 
Table S12 Baseline features of all patients with coronavirus disease 2019 (COVID-19) who started treatment with or without immunoglobin

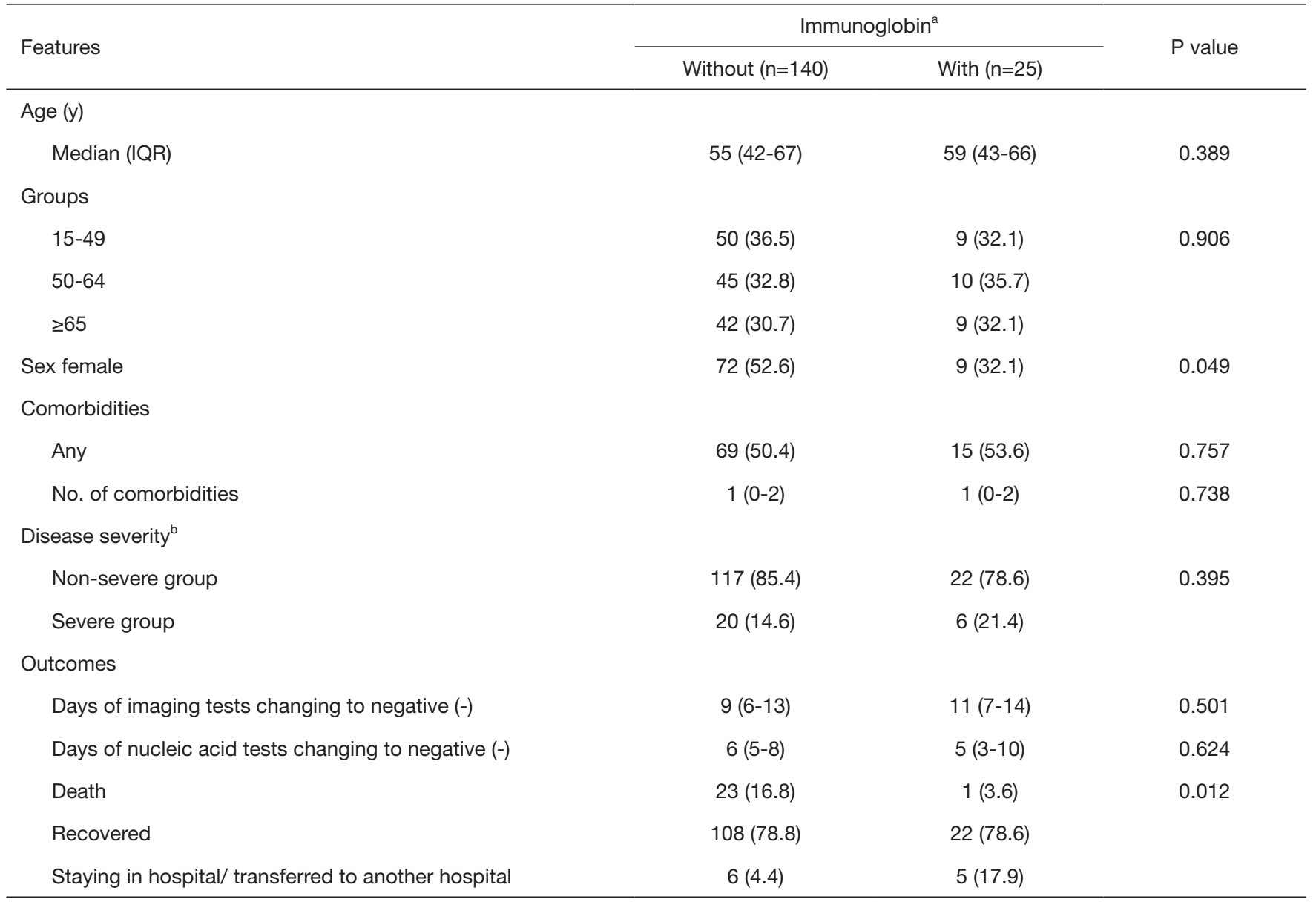

Data are $\mathrm{n}(\%)$ or median (IQR). ${ }^{\text {a }}$, immunoglobin was defined as Anatomical Therapeutic Chemical (ATC) classification codes started with J06BA. ' , the patient's baseline condition was classified into four levels according to the guidelines: mild, general, severe, critical severe respectively, the first two levels were further classified as a non-severe subgroup and the latter two as a severe subgroup. IQR, interquartile range. 


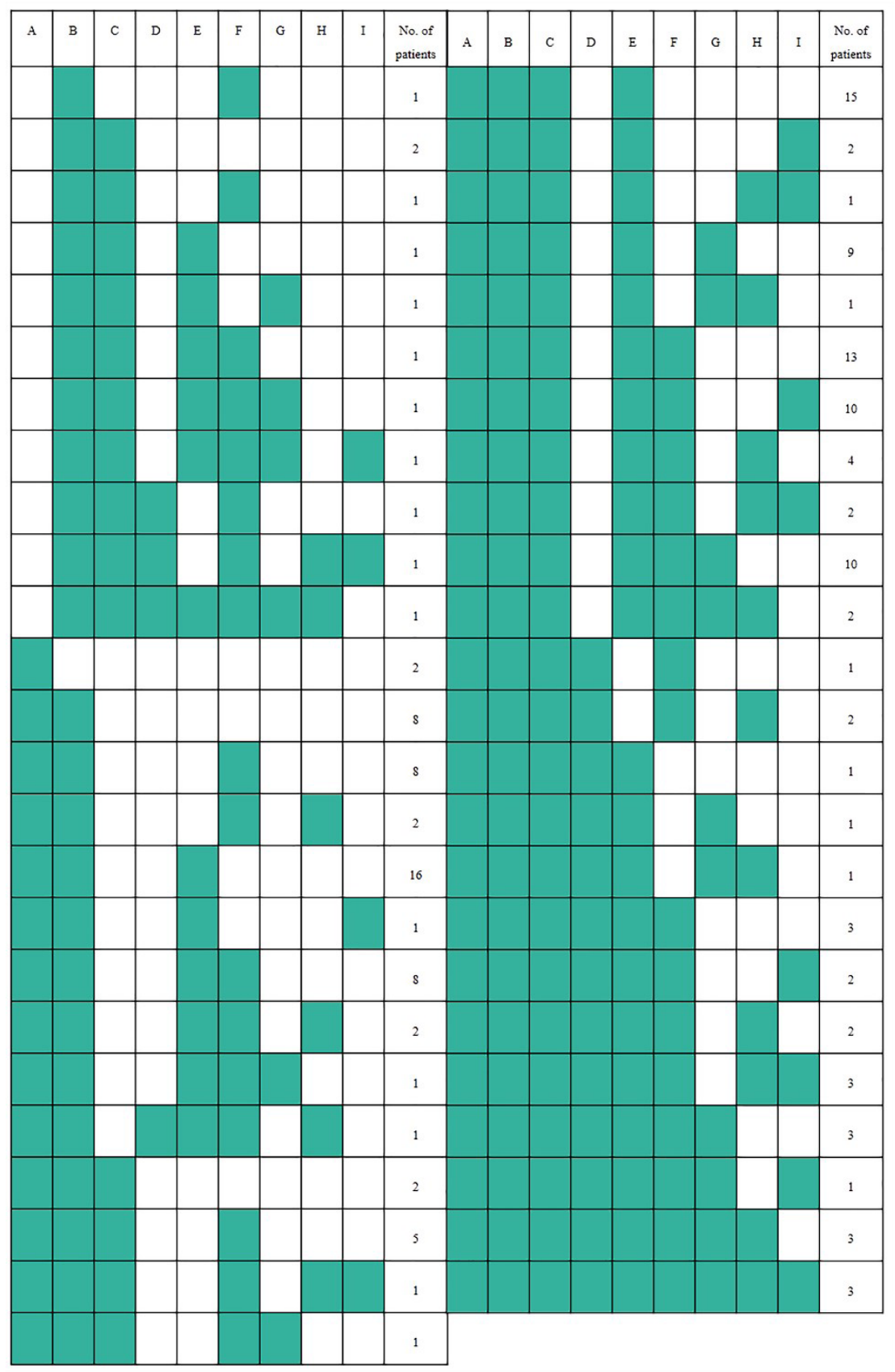

Figure S1 Medication combinations of 165 patients with coronavirus disease 2019 (COVID-19). The column with green color means with that specific medication. A. antivirals [Anatomical Therapeutic Chemical (ATC) classification codes started with J05]. B. antibacterials (J01). C. glucocorticoids (H02AB). D. antimycotics (J02). E. general nutrients (V06). F. traditional Chinese medicine (TCM, identified using drug name). G. vasoactive drugs (C01DA, C01CA, C04AB01). H. intestinal microecological regulators (A07F). I. immunoglobin (J06BA). 


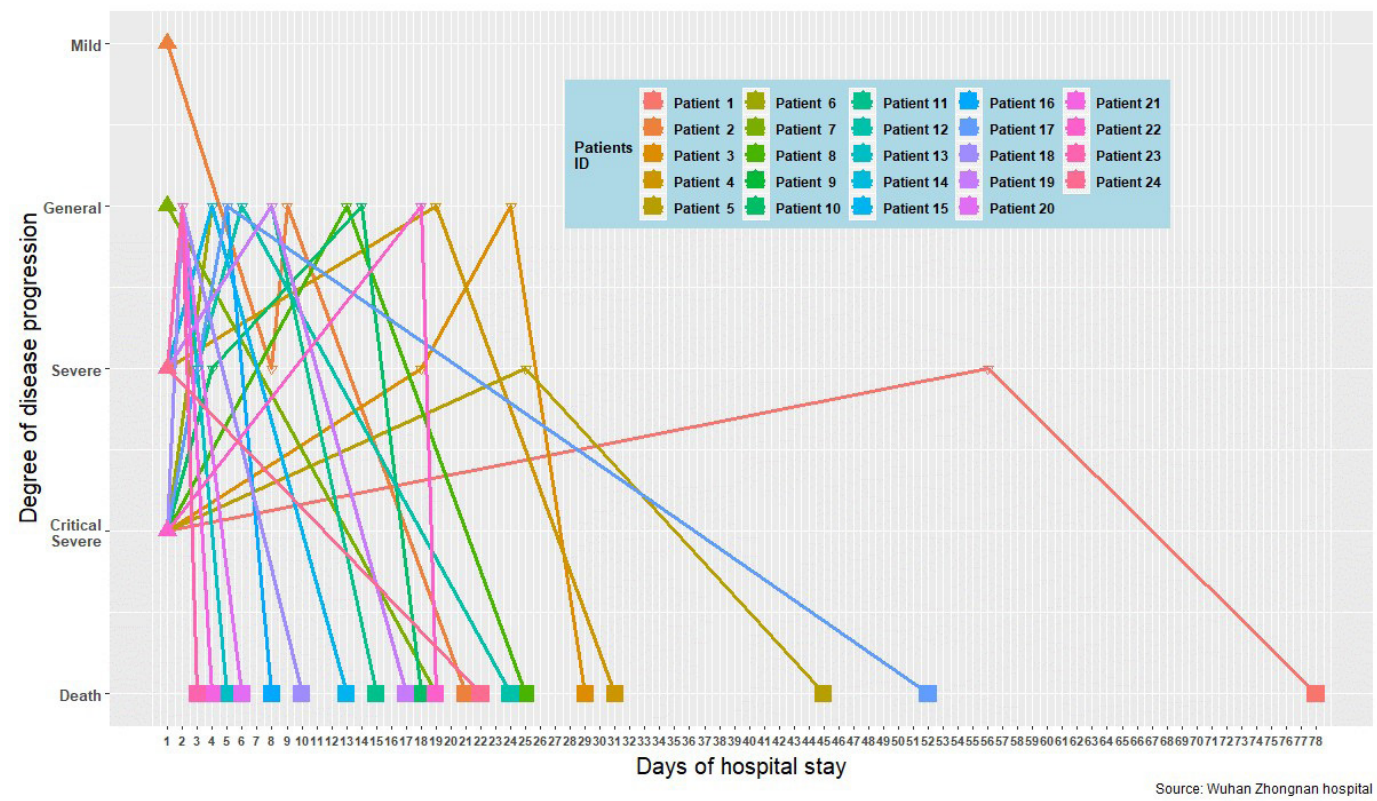

Figure S2 Disease progression of 24 death patients with coronavirus disease 2019 (COVID-19) by days of hospital stay. This figure presented the disease progression of 24 death patients since baseline. The patient's baseline condition was classified into four levels according to the guidelines "Diagnostic and treatment protocol for Novel Coronavirus Pneumonia (trial fifth version)": mild, general, severe, critical severe, respectively. 